\title{
Elastic resistance training is more effective than vitamin D3 supplementation in reducing oxidative stress and strengthen antioxidant enzymes in healthy men
}

\author{
Fardin Kalvandi ${ }^{1}$, Mohammad Ali Azarbayjani ${ }^{2}$, Ronak Azizbeigi $\mathbb{D}^{3}$ and Kamal Azizbeigi (iD ${ }^{1 凶}$ \\ (C) The Author(s), under exclusive licence to Springer Nature Limited 2021
}

OBJECTIVE: Today, reducing oxidative stress and improving the antioxidant system with antioxidant supplements along with exercise training has received a lot of attention. Vitamin D plays a very important role in general health and reducing oxidative stress. The aim of this study was to examine the effect of vitamin D3 supplements during elastic-band resistance training (EBT) on oxidative stress and antioxidant indices in healthy men.

METHODS: Forty healthy men (Serum: $20 \leq 25(\mathrm{OH}) D \leq 25 \mathrm{ng} / \mathrm{mL}$ ) voluntarily participated in the current study and randomly were assigned to EBT-vitamin D3 (ED, $n=10$ ), EBT-placebo (EP, $n=10$ ), vitamin D3 (VD, $n=10)$, and control (Con, $n=10)$. EBT was performed three times per week on non-consecutive days for eight weeks, in seven exercises. The subjects in the ED, VD, and EP consumed 50,000 IU vitamin D3 or placebo once every 2 weeks. Ten ccs blood samples were collected before and after exercise training and the total antioxidant capacity (TAC), superoxide dismutase (SOD), glutathione peroxidase (GPX), and creatine kinase (CK) activities were measured in the plasma. Malondialdehyde (MDA), as the lipid peroxidation index, and 25(OH) D were measured in the plasma.

RESULTS: We found that there was a significant difference between ED with VD $(p=0.011)$ and Con $(p=0.022)$ for MDA. A significant difference was also seen for SOD in ED with VD $(p=0.024)$ and Con $(p=0.038)$ and TAC in ED with VD $(p=0.020)$ and Con $(p=0.030)$, and GPX in ED with VD $(p=0.040)$ and Con $(p=0.010)$. While there were no significant differences between ED and EP in all mentioned variables $(p>0.05)$.

CONCLUSION: Finally, it can be said that elastic resistance training improved antioxidant defence. However, vitamin D3 supplementation during resistance elastic training has no synergistic effect on attenuating oxidative stress indices.

European Journal of Clinical Nutrition (2022) 76:610-615; https://doi.org/10.1038/s41430-021-01000-6

\section{INTRODUCTION}

Reactive oxygen species (ROS), such as superoxide and hydrogen peroxide, are generated in all cells by mitochondrial and enzymatic sources [1]. In aerobic organisms in a resting state, incomplete reduction mitochondria oxygen can lead to ROS [2] and left unchecked, these reactive species can cause oxidative damage to DNA, proteins, and membrane lipids [1]. Also, ROS can cause cell necrosis by cell membrane lipid peroxidation, the oxidation of carbonylated proteins, the decrease of glutathione content, and suppress the antioxidant enzyme such as superoxide dismutase (SOD), glutathione peroxidase (GPX), and total antioxidant capacity (TAC), and all of these finally lead to low cellular lifespan [3].

SOD catalyzes the dismutation of superoxide anion free radical $(\mathrm{O} 2-)$ into molecular oxygen and hydrogen peroxide $(\mathrm{H} 2 \mathrm{O} 2)$ and decreases $\mathrm{O} 2-$ a level that damages the cells at excessive concentration [4], while GPX reduces $\mathrm{H} 2 \mathrm{O} 2$ to water to limit its harmful effects [5]. Both of these mechanisms will reduce oxidative stress and Malondialdehyde (MDA) concentration as a lipid peroxidation factor. Oxidative stress is associated with the ageing process and ageing-related functional disorders, and it has been reported to cause many diseases including cardiovascular disease (CVD), diabetes, chronic obstructive pulmonary disease (COPD), chronic kidney disease, cognitive impairment, and dementia, cancer, and sarcopenia [6].

Various approaches purpose to strengthen the antioxidant system and reduce oxidative stress, and many studies have examined the effect of the different types of exercise training such as resistance training (RT), which increases antioxidant capacity and attenuates oxidative stress $[7,8]$. We previously showed that RT at load progressive mode could decrease oxidative stress and increase antioxidant enzyme activities [9]. However, some studies have reported that RT has less influence on antioxidant adaptation and oxidative stress, judging by some variables $[10,11]$. In, on the

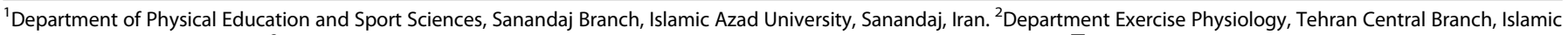
Azad University, Tehran, Iran. ${ }^{3}$ Department of Basic Sciences, Sanandaj Branch, Islamic Azad University, Sanandaj, Iran. ${ }^{凶}$ email: kazizbeigi@gmail.com 
other hand, conventional RT with barbell and dumbbell and also RT machines are expensive. Access to machines requires both facilities and financial resources. Besides these disadvantages, RT with conventional machines and free weight may pose a higher risk of injury or overuse, especially to older people [12]. Also, in some special conditions, such as the COVID-19 pandemic situation, individuals may not be able to attend clubs and public places to use these machines. For this reason, the need to replace RT with weight machines bulk with other less costly, simple training methods is another type of exercise called elastic RT [13].

Elastic resistance devices such as elastic bands are being increasingly used for muscular conditioning for different aims and population types; they are more affordable and more accessible than weight machines $[14,15]$. It has been shown that elastic RT is able to promote similar strength gains to conventional RT, in different population profiles and using diverse protocols [16].

Furthermore, research shows that antioxidant supplements with exercise training serve to increase the effects of synergies of exercise training on the antioxidant capacity system and attenuate oxidative stress [17]. Vitamin D is a group of fat-soluble secosteroids responsible for increasing intestinal absorption of calcium, magnesium, and phosphate, and multiple other biological effects such as antioxidant and anti-inflammatory [18]. Although Vitamin $D$ is a membrane antioxidant with inhibited lipid peroxidation, a few studies were conducted on the effect of exercise training with vitamin D3 supplementation on oxidative stress and antioxidant capacity.

Therefore, the present study was carried out with the aim of investigating the effect of vitamin D3 supplementation during elastic-band resistance training (EBT) on enzyme antioxidant activities and oxidative stress in healthy males.

\section{MATERIALS AND METHODS \\ Subjects}

Forty healthy males with no experience of RT at least in the one year before the current study volunteered to participate in the study and were randomly assigned to EBT-vitamin D3 (ED; $n=10$ ), EBT-placebo (EP; $n=$ $10)$, vitamin $\mathrm{D}(\mathrm{VD} ; n=10)$, and control (Con; $n=10)$ using the online software randomisation [19]. According to the feature of the present study and $p \leq 0.05$ the as significance level, also based on the results of previous studies (standard deviation, sample size of similar studies with similar aims and method), 10 subjects was considered as the sample size in each group. The subjects were asked to refrain from resistance and cardiovascular training during the study. Also, we asked them to minimise recreational physical activity such as swimming, climbing and walking. The participants revealed their medical history, obtained written approval from their physicians, and signed an informed consent form to participate in the study. None of the subjects was taking exogenous anabolic-androgenic steroids, drugs, medication, or dietary supplements with potential redox or inflammatory responses. To reduce the interference of sunlight in the results, the study was done in December, January, and February (2016-2017). The investigation was approved by the committee on the use of human research subjects at the Islamic Azad University of Sanandaj Branch. The subjects attended an information and familiarisation session in which all details of the research procedures were explained. A flow chart of subject enrolment and allocation to the study was presented in Fig. 1.

\section{Primary assessment}

In a session before any intervention, 10 ccs peripheral blood was gathered from the antecubital vein of the left arm in a seated position after 5 min of rest. After that, in the second session, the anthropometric characteristics such as the subjects' height and weight (Seca, Mod 220, Germany) were measured according to the current instruction. The body fat percentage (BF\%) was estimated by Lange skinfold calliper (Beta Technology Incorporated, Cambridge, Massachusetts, USA) in three parts-chest, abdomen, and thigh in standing position-using the equation of Jackson and Pollock [20], and was repeated after the exercise training period.

Then, in order to assess the effect of the elastic-band RT on improving strength and to evaluate the efficacy of the exercise training programme, functional capacity tests were done.

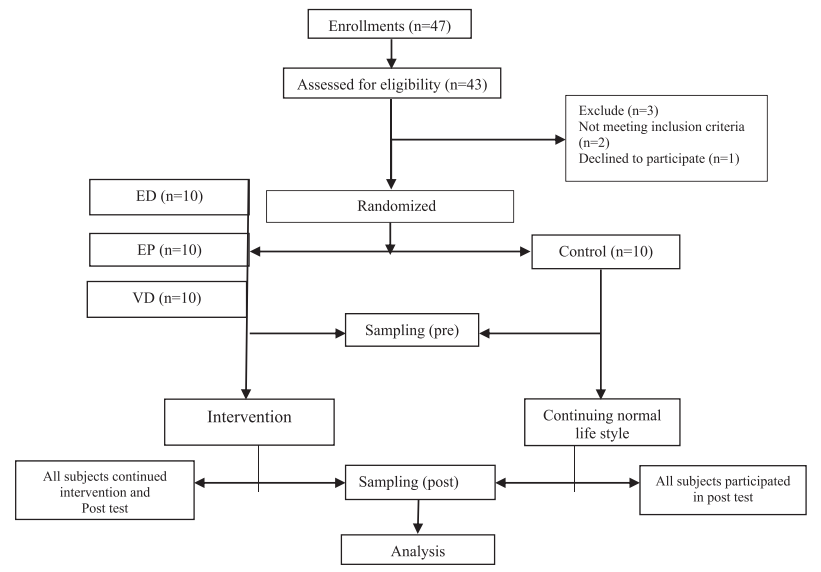

Fig. 1 Flow chart of subject enrollment, allocation, dropout, intervention, and final analysis, $n=4$ excluded from the study because they were not eligible. $n=2$ were excluded due to not meeting inclusion criteria. $n=1$ was excluded because he did not unable to cooperate until the end of the study. Finally, 40 subjects were analyzed in the end of the study.

Table 1. Characteristics and functional variables before starting the study.

\begin{tabular}{|c|c|c|c|c|}
\hline & ED & EP & VD & Control \\
\hline Age (year) & $22.4 \pm 1.2$ & $21.1 \pm 2.1$ & $22.1 \pm 1.1$ & $22.5 \pm 2.4$ \\
\hline Weight (kg) & $73.5 \pm 3.5$ & $71.4 \pm 3.4$ & $72.8 \pm 3.3$ & $71.8 \pm 4.1$ \\
\hline High $(\mathrm{cm})$ & $176.7 \pm 4.7$ & $174.7 \pm 4.8$ & $176.4 \pm 4.7$ & $175.4 \pm 4.1$ \\
\hline BF\% (pre) & $21.9 \pm 1.5$ & $22.1 \pm 1.8$ & $22.9 \pm 0.99$ & $21.7 \pm 1.6$ \\
\hline (post) & $19.7 \pm 1.8$ & $19.8 \pm 0.94$ & $22.3 \pm 0.67$ & $21.4 \pm 1.2$ \\
\hline $\begin{array}{l}\text { Push up test } \\
\text { (no. of } \\
\text { reps) pre }\end{array}$ & $18 \pm 4$ & $17 \pm 6$ & $18 \pm 3$ & $17 \pm 4$ \\
\hline post & $29 \pm 5$ & $27 \pm 5$ & $23 \pm 4$ & $19 \pm 5$ \\
\hline $\begin{array}{l}\text { Squat test } \\
\text { (no. of } \\
\text { reps) pre }\end{array}$ & $27 \pm 6$ & $26 \pm 6$ & $27 \pm 6$ & $25 \pm 5$ \\
\hline post & $38 \pm 5$ & $39 \pm 7$ & $33 \pm 5$ & $27 \pm 6$ \\
\hline
\end{tabular}

$B F \%$ body fat percentage, no. of reps number of repetitions.

A push-up test was applied to access muscular endurance chest and shoulders. This test was done continuously without rest and asked the subjects to do more push up as many as possible. The squat test (without loading) was also used to monitor the subjects' development of lower extremity function. The test requires the subjects to complete as many squats as possible with no rest. The subjects performed a specific warm-up for 10-15 min, with the push-up test always performed before the squat test, and with a minimum recovery period of $10 \mathrm{~min}$ between the 2 tests [21]. These tests were done before intervention (pretest) and were repeated after 8 weeks of elastic training (posttest). The descriptive and functional characteristics of the subjects are presented in Table 1.

\section{Elastic resistance band training}

Subjects were instructed in all exercise techniques by an expert technician. Then the subject was trained in the techniques used for each exercise and then, performed several sets at minimum resistance by an elastic band, and necessary feedback was given to the subjects (Thera-Band ${ }^{\circledR}$, The Hygenic Corporation, Akron, OH, USA; colours-Tan) to ensure that the exercise was being performed correctly. The participants also learned how to control the intensity of the exercises through the scale OMNI-RES.

The elastic RT was performed three times a week on non-consecutive days for eight weeks. Each exercise session consisted of a general warm-up 
Table 2. Exercise training protocol.

\begin{tabular}{|c|c|c|c|c|c|c|c|c|}
\hline Week & 1 & 2 & 3 & 4 & 5 & 6 & 7 & 8 \\
\hline Session/week & 3 & 3 & 3 & 3 & 3 & 3 & 3 & 3 \\
\hline Sets & 3 & 3 & 3 & 3 & 3 & 3 & 4 & 4 \\
\hline Repetition (no. of reps) & 15 & 15 & 10 & 10 & 10 & 10 & 10 & 10 \\
\hline OMNI-RES & $1-7$ & & & $7-8$ & & $8-9$ & $7-9$ & \\
\hline Rest interval between sets (s) & 60 & 60 & $90-120$ & $90-120$ & $90-120$ & $90-120$ & $90-120$ & $90-120$ \\
\hline
\end{tabular}

OMNI-RES OMNI-resistance exercise scale.

Table 3. Nutrient analysis of the dietary records before and after the training and supplementation.

\begin{tabular}{|c|c|c|c|c|c|c|}
\hline & & ED & EP & VD & Control & $P$ \\
\hline \multirow[t]{2}{*}{ Vitamin C (mg/day) } & Pre & $64.1 \pm 23$ & $61.4 \pm 6.7$ & $67.6 \pm 9$ & $72.6 \pm 5.51$ & 0.318 \\
\hline & Post & $73 \pm 12$ & $64.5 \pm 8.8$ & $74 \pm 6.5$ & $73.12 \pm 7.3$ & 0.229 \\
\hline$\alpha$ - tocopherol (mg/day) & Post & $6.6 \pm 1.7$ & $5.2 \pm 0.9$ & $6.5 \pm 1.2$ & $6.1 \pm 1.2$ & 0.06 \\
\hline \multirow[t]{2}{*}{ B- carotene $(\mu \mathrm{g} /$ day $)$} & Pre & $500.3 \pm 393$ & $615.2 \pm 399$ & $620.2 \pm 391$ & $625.8 \pm 248$ & 0.455 \\
\hline & Post & $560.3 \pm 987.2$ & $642.6 \pm 406$ & $640.9 \pm 210$ & $634 \pm 238$ & 0.116 \\
\hline Selenium (mg/day) & Post & $62.9 \pm 13$ & $55.3 \pm 8.3$ & $54.1 \pm 9.9$ & 54. $7 \pm 6.1$ & 0.146 \\
\hline \multirow[t]{2}{*}{ Vitamin D ( $\mu \mathrm{g} /$ day $)$} & Pre & $5.2 \pm 6.4$ & $5.9 \pm 5.8$ & $5.2 \pm 4.9$ & $5.6 \pm 5.5$ & 0.512 \\
\hline & Post & $5.4 \pm 5.8$ & $5.5 \pm 5.1$ & $5.8 \pm 5.2$ & $5.9 \pm 6$ & 0.724 \\
\hline
\end{tabular}

Data expressed as Mean \pm SD.

of $10 \mathrm{~min}$ at the running fashion and dynamic stretching, and finally with 10-15 min of stretching exercises for a cool down at the end of the exercise session [22]. The movements involved were upper body and lower body exercises. The exercises included: Knee flexion (unilateral exercise performed in the prone position), Knee extension (unilateral exercise performed in the chair position), Squat (Standing status). The upper limb exercises consisted of bench flies (Standing status), Overhead press (Standing status), elbow flexion and extension (Standing status), Lat pulldown (Standing status), and Trunk Curl-up (performed in the prone position). All training sessions were performed under the supervision of a qualified and experienced instructor. The subjects were asked to perform exercises at the full range of motion at moderate to high velocity as possible [23]. To control the intensity of training, the OMNI-RES was always visible during each training session for all subjects. Following an adaptation phase of 2 weeks using low resistance (yellow Thera-Band ${ }^{\circledR}$, 3 sets of 10-15 repetitions per exercise) exercise intensity was progressively increased by adapting the resistance of the elastic band (based on the Thera-Band ${ }^{\circledR}$ force-elongation table) [13], from yellow to green, silver, or even and further to gold colour. The rate of progression was based on individual improvements, band colour was changed if the participant would have been able to perform two more repetitions in the second set and reported to be below seven on the OMNI Resistance for active muscle scale ( 0 extremely easy to 10 extremely hard) [21]. It should be noted that 10-15 repetitions were maintained for the 8-week elastic RT, with three sets during the 8 weeks. The control group was asked to refrain from any RT and maintain their normal physical activity and nutrition behaviours for the study period. Exercise training details were present in Table 2.

\section{A controlled diet and vitamin D3 supplementation}

The subjects were asked not to modify their diet for the duration of the study, except for refraining from consuming any products containing high levels of antioxidants, and anti-inflammatory drugs. The subjects also filled out a two-day dietary recall before and after the treatment (Table 3). The daily dietary intake of vitamins $D, C, E$, beta-carotene, and selenium was estimated using a software programme (Esha Research, Salem, OR, USA). Also, to limit the interfering effect of skin-synthesised vitamin $D$, the present study was conducted at the end of the autumn and early winter season, and subjects were advised to reduce traffic as much as possible from 10 a.m. to 3 p.m. All subjects in the ED and VD consumed vitamin D3 capsules $(50,000$ IU, Zahravi, Iran), every two weeks. A capsule $(500 \mathrm{mg}$ sucrose, after breakfast) was taken every two weeks by the EP. The capsules (Vitamin D3 and placebo) were identical in appearance (i.e., size, shape, and colour). The timing of the intakes was standardised to avoid possible interference with the results, and the dosage was carefully monitored. On this basis, none of the subjects was aware of the capsules contents. Also, the only data analyzer did not know about the grouping of subjects and was blind.

\section{Blood sampling and biochemical assays}

Before any intervention, a 10 ccs blood sample was obtained from the antecubital vein of the left hand. The blood sampling was repeated $72 \mathrm{~h}$ after the last session of the training. The blood samples were centrifuged at 2200-2500 rpm for $10 \mathrm{~min}$ and plasma and the surface layer were separated from haematocrits. The plasma samples were used for the measurement of TAC, MDA, SOD, and GPX and Creatine Kinase (CK), and 25(OH)D.

The SOD and GPX activity was measured by using a commercially available kit (Randox Laboratories Ltd., Crumlin, Country Antrim, UK), and were expressed in U/L. Similarly, a commercially available kit (Cat. No. NX 2332, Randox, Crumlin, UK) was used for the measurement of the antioxidant capacity in the plasma (TAC). The plasma MDA was measured based on the method of Buege and Aust [24]. The plasma CK activity was tested using a standard kit (Pars Azmoon, Iran) and enzyme activity was expressed as $\mathrm{U} / \mathrm{L}$.

\section{Statistical analyses}

All values were reported as mean \pm SD. First, the normal distribution of data was confirmed with the Kolmogorov-Smirnov test. The homogeneity of the physical characteristics of the subjects at the beginning of the study was tested with one-way ANOVA. Also, one-way ANOVA was used to compare the results of the dietary survey. The data regarding the biochemical parameters were compared using repeated measures (preand post-training) ANOVA to investigate the influence of time and the training intervention. Where a significant main effect and/or interaction was observed, the Tukey post hoc test was applied to determine the source of significance. Partial eta-squared (np2) was used to determine the effect size of training, vitamin D3 and training, vitamin D3 interaction. 
Table 4. Changes variable before and after training and supplementation.

\begin{tabular}{|c|c|c|c|c|c|c|}
\hline Variables & & ED & EP & VD & Control & Time $\times$ group $(p)$ \\
\hline \multirow[t]{2}{*}{ MDA $(\mathrm{nmol} / \mathrm{ml})$} & Pre & $1.91 \pm 0.53$ & $2.04 \pm 0.45$ & $1.96 \pm 0.6$ & $1.92 \pm 0.52$ & 0.004 \\
\hline & Post & $1.07 \pm 0.30$ & $1.27 \pm 0.36$ & $1.75 \pm 0.54$ & $1.90 \pm 0.46$ & \\
\hline \multirow[t]{2}{*}{ SOD (U/I) } & Pre & $188.2 \pm 25.3$ & $180.6 \pm 20.5$ & $176.6 \pm 24.5$ & $188.7 \pm 17.3$ & 0.001 \\
\hline & Post & $214 \pm 21.4$ & $209.3 \pm 17.3$ & $179.2 \pm 22.6$ & $171.5 \pm 19.9$ & \\
\hline \multirow[t]{2}{*}{ TAC (mmol/l) } & Pre & $1.28 \pm 0.15$ & $1.35 \pm 0.24$ & $1.26 \pm 0.17$ & $1.32 \pm 0.24$ & 0.02 \\
\hline & Post & $1.65 \pm 0.38$ & $1.53 \pm 0.19$ & $1.37 \pm 0.25$ & $1.28 \pm 0.15$ & \\
\hline & Post & $6194 \pm 1472.4$ & $5971.8 \pm 1070$ & $4404.6 \pm 926.9$ & $4209.5 \pm 588.4$ & \\
\hline \multirow[t]{2}{*}{$\mathrm{CK}(\mathrm{U} / \mathrm{l})$} & Pre & $119.1 \pm 14.9$ & $122 \pm 25.1$ & $121 \pm 25.1$ & $123.8 \pm 19.4$ & 0.7 \\
\hline & Post & $135.8 \pm 33.5$ & $137.16 \pm 13.2$ & $123.1 \pm 24.6$ & $125.9 \pm 11.1$ & \\
\hline \multirow[t]{2}{*}{$25(\mathrm{OH}) \mathrm{D}(\mathrm{ng} / \mathrm{mL})$} & Pre & $20.4 \pm 4.5$ & $21.2 \pm 4.5$ & $22.5 \pm 3.4$ & $21.2 \pm 4.9$ & 0.001 \\
\hline & Post & $45.51 \pm 6.9$ & $22.4 \pm 7.1$ & $44.2 \pm 9.4$ & $22.7 \pm 6.1$ & \\
\hline
\end{tabular}

The data are presented as Mean \pm SD.

$M D A$ malondialdehyde, $S O D$ superoxide dismutase, $T A C$ total antioxidant capacity, GPX glutathione peroxidase, $C K$ creatine kinase, 25(OH)D 25-hydroxy vitamin D.

The Cohen's D test was used to estimate the effect size between preand post-training in each group; a value of less than 0.2 indicated a negligible effect size, between 0.2 and 0.5 a small effect size, between 0.5 and 0.8 a moderate effect size, and greater than 0.8 a large effect size. The data analysis was performed by using SPSS for WINDOWS software version 19. A $p$-value of $p \leq 0.05$ was used as the level of significance.

\section{RESULTS}

The results showed no significant difference in total antioxidants between the four groups $(p>0.05)$. The antioxidant analyses of the dietary records of the subjects before and after the elastic RT and supplementation period are presented in Table 3.

We found that elastic training increased push up tests in ED [pre: 18 vs. post 29 reps; $(p \leq 0.001)$ ] and EP [pre: 17 vs. 27 reps; $(p \leq 0.001)]$ by $61 \%$ and $58.8 \%$, respectively.

Also, the squat test was increased significantly in ED [pre: 27 vs 38 reps ( $p \leq 0.001)$ ] and EP [pre: 26 vs 39 reps ( $p \leq 0.001)$ ] by $40.7 \%$ and $50 \%$, respectively. However, there is no significant difference between ED and EP for the mentioned variables. Also, push up and squat test changes in the VD and control groups was insignificant $(p>0.05)$.

Body fat percentage (BF\%) was significantly decreased in the ED $[-10.5 \% ; p=0.003 ;]$, EP $[-10.4 \% ; p=0.001 ;(0.28)]$, and VD $[-2.62 \% ; p=0.024 ;$, while there was a significantly in the ED and EP than VD and Con $(p \leq 0.05)$.

The results showed that there is a significant difference in time, the main effect of elastic RT, and vitamin D3 supplementation for MDA $(p=0.001, n p 2=0.437), \mathrm{CK}(p=$ $0.005, \mathrm{np} 2=0.334)$, SOD $(p=0.049, \mathrm{np} 2=0.112), \mathrm{GPX}(p=0.001$, $\eta \mathrm{p} 2=0.125)$, and TAC $(p=0.010, \mathrm{np} 2=0.185)$ after training or supplementation.

We found that the MDA significantly decrease in the ED $(-43.98 \%, p=0.001, d=0.713)$ and $\operatorname{EP}(-37.75 \%, p=0.001, d=$ $0.701)$. The SOD $(13.71 \%, p=0.023, d=0.454), \operatorname{GPX}(44 \%, p=$ $0.002, d=0.654)$, and TAC (28.91\%, $p=0.022, d=0.501)$ significantly increased in ED in the post-test. Also, in the EP, SOD ( $p=$ $0.003, d=0.622), \operatorname{GPX}(p=0.002, d=0.379)$ and TAC $(p=0.034$, $d=0.324$ ) significantly increased by $15.89 \%, 30.24 \%$, and $13.33 \%$, respectively.

Also, the time $\times$ groups interaction was significant in MDA ( $p=$ $0.006, n p 2=0.313)$, SOD $(p=0.007, n p 2=0.299), \operatorname{GPX}(p=0.001$, $\eta p 2=0.113)$, and TAC $(p=0.048, \eta p 2=0.211)$. We found that there were significant differences between ED and VD $(p=0.040)$, and ED with Con $(p=0.019)$ for MDA.
Furthermore, there is a significant difference in SOD [ED with VD $(p=0.003)$, and ED with Con $(p=0.007)$; EP with VD $(p=0.023)]$ and TAC [ED with VD $(p=0.028)$, and ED with Con $(p=0.040)$ ], and GPX [ED with VD $(p=0.006)$, and ED with Con $(p=0.003)$; EP with VD $(p=0.002)$, EP with Con $(p=0.001)]$.

It was observed that supplementation with vitamin D3 increased the $25(\mathrm{OH}) \mathrm{D}$ in the plasma by $(123 \% p=0.001, d=$ $0.969)$ and $(96.4 \% p=0.001, d=0.960)$ in the ED and VD, respectively.

There is a significant difference between groups [ED with EP and control $(p=0.001)$, VD with Con $(p=0.001)]$ see Table 4.

\section{DISCUSSION}

The aim of the present study was to investigate the effect of vitamin D3 supplementation during elastic training, as a simple and inexpensive training method, on MDA and antioxidant enzymes in healthy men.

Overall, we found that although vitamin D3 supplementation had a positive effect in attenuating oxidative stress, the effect of elastic training is greater and more effective in this issue.

The results showed that the push-up and squat tests improved significantly in ED and EP. It implies the elastic RT programme was effective in functional and physiological features. Ageing, inactivity, and disuse are associated with a decline in muscle mass, structure, and strength [25]. It has been reported that elastic RT is able to promote similar strength gains to conventional RT, in different population profiles and using diverse protocols [16]. Therefore, it seems that elastic RT could be used as a rehabilitation strategy in the elderly and clinical conditions.

The prevalence of obesity has increased worldwide reaching pandemic levels [26]. We found that body fat percent (BF\%) was affected by elastic training. On the other hand; adipose tissue may increase oxidative stress [27]. Therefore, BF\% decrement may have had a positive effect on oxidative stress and antioxidant variables behaviour in the current study. Thus, today elastic RT could be used as an inexpensive and simple approach to reduce body fat and body weight management.

The results showed that SOD and GPX activity increased significantly in ED and EP compared to the VD and the control group. It can be said that the influence of elastic training was greater and more crucial in increasing the antioxidant enzyme activities. It is known that exercise-induced adaptation of antioxidants is dependent on several variables, which include tissue, age, duration, intensity, and specific exercise mode [28]. 
In the present study, we used low to moderate intensity, while most studies have investigated the effects of moderate to highintensity RT $[9,29]$. It seems that the present protocol of elastic RT with low intensity could challenge the physiological haemostasis to increase antioxidant activity such as SOD and GPX because it has been reported that resistance exercise at 30\% 1RM (similar intensity to the present study) can increase free radical generation to stimulate antioxidant adaptation [30].

Also, we found that the combination of vitamin D3 with elastic training has low effects of synergies. It has been reported that vitamin $D$ is an antioxidant vitamin; it can prevent iron-dependent lipid peroxidation in the cell membrane [31]. It should be mentioned that few investigations have conducted the effect of vitamin D supplementation with exercise training on antioxidant enzyme activity; therefore, the explanation of behaviour and changes in enzyme activities is difficult to proffer. It seems that antioxidant supplementation alone has little effect on antioxidant enzymes activity such as SOD and GPX. In accordance with the findings of the present study, it has been reported that in a rat model, the combination of exercise training and antioxidant supplementation increased the antioxidant enzyme activities (GPX, CAT) compared with each individual intervention, while antioxidant supplementation had no significant effect $(p>0.05)$ on activities of antioxidant enzymes in sedentary animals [32].

The results showed that MDA concentration decreased significantly in the ED and EP groups, compared to the VD and the control groups, while While the TAC increased only in the ED and EP. This means that only elastic training had an effect on these changes. MDA can be measured in plasma and is used for the purpose of estimating lipid-specific oxidative stress [33]. It has been mentioned that lipid peroxidation-related adaptations after RT have been poorly investigated and the available studies on the effect of resistance exercise on oxidative stress report contradictory findings $[9,34,35]$. In accordance with the findings of the present study, it has been reported that RT could decrease MDA concentration following RT [29]. It seems that TAC, SOD, and GPX improvement could decrease MDA concentration in the present study. However, vitamin D3 supplementation could not affect lipid peroxidation. Recently, consuming antioxidants such as vitamin D have increased due to reports suggesting that vitamin $D$ deficiency exists in some athletes, particularly those who participate in indoor sports such as bodybuilding [36]. However, we found that vitamin D3 supplementation had no effect on oxidative stress markers. Our subjects were healthy men with normal body fat percentages. It seems that oxidative stress and existing free radicals may interfere in this issue because oxidative stress results in the oxidative catabolism of $25(\mathrm{OH}) \mathrm{D}$ [37]. On the other hand, low vitamin D3 dose may affect present results. We used 50,000 IU vitamin D3 for each subject, once every two weeks, which means 3600 IU vitamin D3 per day, approximately. Before starting the supplementation, we found that level $25(\mathrm{OH}) \mathrm{D}$ basal was low and it reached $45 \mathrm{ng} / \mathrm{mL}$ after the supplementation. Some authors recommend much higher initial dosages if $25(\mathrm{OH}) \mathrm{D}$ levels are deficient, ranging from 2000 to 200,000 IU to maintain optimal $25(\mathrm{OH}) \mathrm{D}$ status [37]. Also, the consumption instruction of vitamin $D$ (daily vs. weekly) may interfere with the function of this vitamin in attenuating oxidative stress. It has been shown that a daily dose of the vitamin may be superior in raising $25(\mathrm{OH}) \mathrm{D}$ levels when compared to a weekly dose [38].

\section{CONCLUSION}

We found that vitamin D3 supplementation during resistance elastic training had no synergistic effect on attenuating oxidative stress indices. We observed that the effect of elastic RT was greater and more prominent in decreasing oxidative stress and in the improvement of the defence antioxidant system.

\section{REFERENCES}

1. Tejero J, Shiva S, Gladwin MT. Sources of vascular nitric oxide and reactive oxygen species and their regulation. Physiol Rev. 2019;99:311-79. https://doi.org/ 10.1152/physrev.00036.2017.

2. Li X, Fang P, Mai J, Choi ET, Wang H, Yang XF. Targeting mitochondrial reactive oxygen species as novel therapy for inflammatory diseases and cancers. J Hematol Oncol. 2013;6:19. https://doi.org/10.1186/1756-8722-6-19.

3. Silva JP, Coutinho OP. Free radicals in the regulation of damage and cell deathbasic mechanisms and prevention. Drug Discov Ther. 2010;4:144-67.

4. Yasui K, Baba A. Therapeutic potential of superoxide dismutase (SOD) for resolution of inflammation. Inflamm Res. 2006;55:359-63.

5. Lubos E, Loscalzo J, Handy DE. Glutathione peroxidase-1 in health and disease: from molecular mechanisms to therapeutic opportunities. Antioxid Redox Signal. 2011;15:1957-97. https://doi.org/10.1089/ars.2010.3586

6. Liguori I, Russo G, Curcio F, Bulli G, Aran L, Della-Morte D. et al. Oxidative stress, aging, and diseases. Clin Interv Aging. 2018;26:757-72. https://doi.org/10.2147/ CIA.S158513.

7. Da Palma RK, Moraes-Silva IC, da Silva Dias D, Shimojo GL, Conti FF, Bernardes N. et al. Resistance or aerobic training decreases blood pressure and improves cardiovascular autonomic control and oxidative stress in hypertensive menopausal rats. J Appl Physiol. 2016;121:1032-8. https://doi.org/10.1152/ japplphysiol.00130.2016.

8. Azizbeigi K, Stannard SR, Atashak S. Green tea supplementation during resistance training minimally affects systemic inflammation and oxidative stress indices in obese men. Jundishapur J Nat Pharm Prod. 2019;14:e61419 https://doi.org/ 10.5812/jjnpp.61419

9. Azizbeigi K, Azarbayjani MA, Peeri M, Agha-alinejad H, Stannard S. The effect of progressive resistance training on oxidative stress and antioxidant enzyme activity in erythrocytes in untrained men. Int J Sport Nutr Exerc Metab. 2013;23:230-8. https://doi.org/10.1123/ijsnem.23.3.230

10. Parise G, Brose AN, Tarnopolsky MA. Resistance exercise training decreases oxidative damage to DNA and increases cytochrome oxidase activity in older adults. Exp Gerontol. 2005;40:173-80. https://doi.org/10.1016/j.exger.2004.09.002

11. Vincent HK, Bourguignon C, Vincent KR. Resistance training lowers exerciseinduced oxidative stress and homocysteine levels in overweight and obese older adults. Obesity. 2006;14:1921-30. https://doi.org/10.1038/oby.2006.224

12. Liu CJ, Latham $\mathrm{N}$. Adverse events reported in progressive resistance strength training trials in older adults: 2 sides of a coin. Arch Phys Med Rehabil. 2010;91:1471-3. https://doi.org/10.1016/j.apmr.2010.06.001

13. Page $P$, Ellenbecker TS. Strength band training. Human kinetics, Leeds. 2011.

14. Thomas $M$, Mueller T, Busse MW. Quantification of tension in thera-band and cando tubing at different strains and starting lengths. J Sports Med Phys Fit. 2005;45:188-98.

15. Hostler DC, Schwirian I, Campos G, Toma K, Crill MT, Hagerman GR, et al. Skeletal muscle adaptations in elastic resistance-trained young men and women. Eur J Appl Physiol. 2001;86:112-8. https://doi.org/10.1007/s004210100495

16. Lopes J, Machado AF, Micheletti JK, de Almeida AC, Cavina AP, Pastre CM. Effects of training with elastic resistance versus conventional resistance on muscular strength: a systematic review and meta-analysis. SAGE Open Med. 2019;7:2050312119831116 https://doi.org/10.1177/2050312119831116

17. Yavari A, Javadi M, Mirmiran P, Bahadoran Z. Exercise-induced oxidative stress and dietary antioxidants. Asian J Sports Med. 2015;6:e24898 https://doi.org/ 10.5812/asjsm.24898

18. Holick F. Sunlight and vitamin D for bone health and prevention of autoimmune diseases, cancers, and cardiovascular disease. Am J Clin Nutr. 2004;80:1678S-88S. https://doi.org/10.1093/ajcn/80.6.1678S. (6 Suppl)

19. Kassambara A. rstatix: pipe-friendly framework for basic statistical tests. 2020 . https://rpkgs.datanovia.com/rstatix/.

20. Jackson AS, Pollock ML. Practical assessment of body composition. Phys Sportsmed. 1985;13:76-90. https://doi.org/10.1080/00913847.1985.11708790

21. Colado JC, Triplett NT. Effect of a short-term resistance program using elastic band versus weight machines for sedentary meddle-age women. J Strength Cond Res. 2008;22:1441-8.

22. Bavaresco Gambassi B, Lopes Dos Santos MD, Furtado Almeida FJ. Basic guide for the application of the main variables of resistance training in elderly. Aging Clin Exp Res. 2019;31:1019-20. https://doi.org/10.1007/s40520-019-01181-y

23. Janusevicius D, Snieckus A, Skurvydas A, Silinskas V, Trinkunas E, Cadefau JA, et al. Effects of high velocity elastic band versus heavy resistance training on hamstring strength, activation, and sprint running performance. J Sports Sci Med. 2017;16:239-46.

24. Buege JA, Aust SD. Microsomal lipid peroxidation. Methods Enzymol. 1978;52:302-10.

25. Seene $T$, Kaasik $P$, Riso EM. Review on aging, unloading and reloading: changes in skeletal muscle quantity and quality. Arch Gerontol Geriatr. 2012;54:374-80. https://doi.org/10.1016/j.archger.2011.05.002 
26. Blüher M. Obesity: global epidemiology and pathogenesis. Nat Rev Endocrinol. 2019;15:288-98. https://doi.org/10.1038/s41574-019-0176-8

27. Sakurai T, Ogasawara J, Shirato K, Izawa T, Oh-Ishi S, Ishibashi Y, et al. Exercise training attenuates the dysregulated expression of adipokines and oxidative stress in white adipose tissue. Oxid Med Cell. 2017;2017:9410954. https://doi.org/ $10.1155 / 2017 / 9410954$.

28. Sallam N, Laher I. Exercise modulates oxidative stress and inflammation in aging and cardiovascular diseases. Oxid Med Cell Longev. 2016:7239639. https://doi. org/10.1155/2016/7239639

29. Azizbeigi K, Azarbayjani MA, Atashak S, Stannard SR. Effect of moderate and high resistance training intensity on indices of inflammatory and oxidative stress. Res. Sports Med. 2015;23:73-87. https://doi.org/10.1080/15438627.2014.975807

30. Güzel NA, Hazar S, Erbas D. Effects of different resistance exercise protocols on nitric oxide, lipid peroxidation and creatine kinase activity in sedentary males. J Sports Sci Med. 2007;6:417-22.

31. Wiseman H. Vitamin $D$ is a membrane antioxidant. Ability to inhibit irondependent lipid peroxidation in liposomes compared to cholesterol, ergosterol and tamoxifen and relevance to anticancer action. FEBS Lett. 1993;326:285-8. 12

32. Marsh SA, Laursen PB, Coombes JS. Effects of antioxidant supplementation and exercise training on erythrocyte antioxidant enzymes. Int J Vitam Nutr Res. 2006;76:324-31.

33. Ito $F$, Sono $Y$, Ito T. Measurement and clinical significance of lipid peroxidation as a biomarker of oxidative stress: oxidative stress in diabetes, atherosclerosis, and chronic inflammation. Antioxidants. 2019;8:72 https://doi.org/10.3390/ antiox8030072. Published 2019 Mar 25

34. Ramel A, Wagner $\mathrm{KH}$, Elmadfa I. Plasma antioxidants and lipid oxidation after submaximal resistance exercise in men. Eur J Clin Nutr. 2004;43:2-6. https://doi. org/10.1007/s00394-004-0432-z

35. Hoffman JR, Im J, Kang J, Maresh CM, Kraemer WJ, French D, et al. Composition lowand high- intensity resistance exercise on lipid peroxidation: role of muscle oxygenation. J Strength Cond Res. 2007;21:118-22. https://doi.org/10.1519/00124278200702000-00022

36. Halliday T, Peterson N, Thomas J, Kleppinger K, Hollis B, Larson-Meyer D. Vitamin $\mathrm{D}$ status relative to diet, lifestyle, injury and illness in college athletes. Med Sci Sports Exerc. 2011;43:335-43. https://doi.org/10.1249/MSS.0b013e3181eb9d4d

37. Cannell JJ, Hollis BW. Use of vitamin D in clinical practice. Alter Med Rev. 2008;13:6-20. PMID: 18377099
38. Close GL, Russel J, Cobley JN, Owens DJ, Wilson G, Gregson W, et al. Assessment of vitamin $D$ concentration in non-supplemented professional athletes and healthy adults during the winter months in the UK: Implications for skeletal muscle function. J Sports Sci. 2013;31:344-53. https://doi.org/10.1080/ 02640414.2012 .733822

\section{ACKNOWLEDGEMENTS}

The authors wish to thank all the volunteers for their enthusiastic participation in this study.

\section{AUTHOR CONTRIBUTIONS}

FK designed the research and collected the data. KA designed the research, supervised the work, and wrote the article. MAA analysed data. RA contributed to the design and implementation of the research.

\section{FUNDING}

This research did not receive any specific grant from funding agencies in the public, commercial, or not-for-profit sectors. All sources of funding are provided by the authors.

\section{COMPETING INTERESTS}

The authors declare no competing interests.

\section{ADDITIONAL INFORMATION}

Correspondence and requests for materials should be addressed to Kamal Azizbeigi. Reprints and permission information is available at http://www.nature.com/ reprints

Publisher's note Springer Nature remains neutral with regard to jurisdictional claims in published maps and institutional affiliations. 